\title{
Longitudinal dependence of characteristics of low-latitude Pi2 pulsations observed at Kakioka and Hermanus
}

\author{
M. Nosé ${ }^{1}$, K. Liou ${ }^{2}$, and P. R. Sutcliffe ${ }^{3}$ \\ ${ }^{1}$ Data Analysis Center for Geomagnetism and Space Magnetism, Graduate School of Science, Kyoto University, Kyoto, Japan \\ ${ }^{2}$ Applied Physics Laboratory, Johns Hopkins University, Laurel, Maryland, USA \\ ${ }^{3}$ Hermanus Magnetic Observatory, South Africa
}

(Received May 6, 2005; Revised August 15, 2005; Accepted August 18, 2005; Online published June 2, 2006)

\begin{abstract}
We statistically investigated longitudinal dependence of characteristics of low-latitude $\mathrm{Pi} 2$ pulsations to find the longitudinal structure of the plasmaspheric cavity mode. We used the geomagnetic field data from two ground stations, Kakioka $\left(27.2^{\circ}\right.$ geomagnetic latitude, 208.5 geomagnetic longitude) and Hermanus $\left(-33.9^{\circ}\right.$ geomagnetic latitude, $82.2^{\circ}$ geomagnetic longitude), and auroral image data acquired by the ultraviolet imager onboard the Polar satellite for the period of December 4, 1996 to March 3, 1997. Our findings include the following: (1) Pi2 amplitude is the largest around the magnetic local time of the auroral breakup site and decreases away from it; (2) when a nightside Pi2 pulsation has large amplitude, a dayside Pi2 pulsation can be observed with a similar waveform; (3) Pi2 pulsations generally have no clear phase differences (mean phase difference of $3.3^{\circ}$ ) between Kakioka and Hermanus, except for some events; and (4) the phase difference is independent on $\triangle$ MLT (difference of magnetic local time between a station and the auroral breakup). These observations suggest that the plasmaspheric cavity mode can be excited globally with a very small value of the azimuthal wave number $(m \approx 0)$.
\end{abstract}

Key words: Pi2 pulsation, plasmaspheric cavity mode.

\section{Introduction}

The plasmaspheric cavity mode has been proposed for an excitation mechanism of $\mathrm{Pi} 2$ pulsations (damped oscillations of the geomagnetic field with a period of 40-150 s) observed at low- and mid-latitude ground stations. This mode is established by fast mode waves that are emitted at a substorm onset and form the standing oscillation within the plasmasphere. Therefore satellites in the low $L$ shell (mostly less than $L \sim 5$ ) observe the geomagnetic field oscillations predominantly in the compressional and radial components when substorms occur (Lin and Cahill, 1975; Sakurai and McPherron, 1983; Takahashi et al., 1995, 1999a; Keiling et al., 2001; Kim et al., 2001). Polar orbiting satellites in low Earth orbit (450-850 km altitude) also detect compressional oscillations that are correlated with lowlatitude Pi2 pulsations on the nightside (Takahashi et al., 1999b; Sutcliffe and Lühr, 2003; Han et al., 2004). Studies that compare magnetometer data from low-latitude ground stations and the CRRES satellite staying inside the plasmasphere revealed radial structure of the fundamental plasmaspheric cavity mode on the nightside (Takahashi et al., 2001, 2003a). Even the radial structure of the second harmonic cavity mode was identified near midnight by Takahashi et al. (2003b). On the morningside (0600-1000 magnetic local time (MLT)), Nosé et al. (2003) examined data from the ETS-VI and EXOS-D satellites as well as ground stations,

Copyright (c) The Society of Geomagnetism and Earth, Planetary and Space Sciences (SGEPSS); The Seismological Society of Japan; The Volcanological Society of Japan; The Geodetic Society of Japan; The Japanese Society for Planetary Sciences; TERRAPUB and confirmed the similar radial structure of the fundamental cavity mode.

As mentioned above, the radial structure of the plasmaspheric cavity mode has been well described by a number of recent studies, though its longitudinal structure is yet to be understood. According to the review paper by Yumoto (1986), nightside Pi2 pulsations at low-latitudes ( $L=1.15-$ 1.83 ) propagate westward in the premidnight and eastward in the postmidnight. Apparent propagating speeds in the longitudinal direction are $\sim 5 \%$, corresponding to the azimuthal wave number $(m)$ of $\sim 1$ if the period of pulsation $(T)$ is $\sim 70 \mathrm{~s}$. Li et al. (1998) reported that $\mathrm{Pi} 2$ pulsations at low-latitudes $(L \sim 1.5-1.6)$ have $|m|<3$ with a dominant westward propagation. Liou et al. (2000) estimated the propagation speed of $\mathrm{Pi} 2$ pulsations at low-latitude $(L \sim 1.3)$ to be $\sim 2.3^{\circ} / \mathrm{s}(m \sim 2$ for $T \sim 70 \mathrm{~s})$. On the other hand, Kitamura et al. (1988) found a very small number of $m(\ll 1)$ for equatorial and low-latitude $(L<1.3) \mathrm{Pi} 2$ pulsations. Nosé et al. (2003) showed an event of low-latitude $(L<1.5) \mathrm{Pi} 2$ pulsation having no phase delay among three local time sectors (0200 MLT, 0700 MLT, and 1500 MLT), suggesting $m \sim 0$. The $m$-number of low-latitude $\mathrm{Pi} 2$ pulsations (i.e., the longitudinal structure of the plasmaspheric cavity mode) is still a controversial issue.

In this study we statistically investigate longitudinal dependence of characteristics of low-latitude $\mathrm{Pi} 2$ pulsations, using ground data and auroral images from the Polar spacecraft, in order to deduce longitudinal structure of the plasmaspheric cavity mode. The ground stations used here are Kakioka in Japan and Hermanus in South Africa, which are 


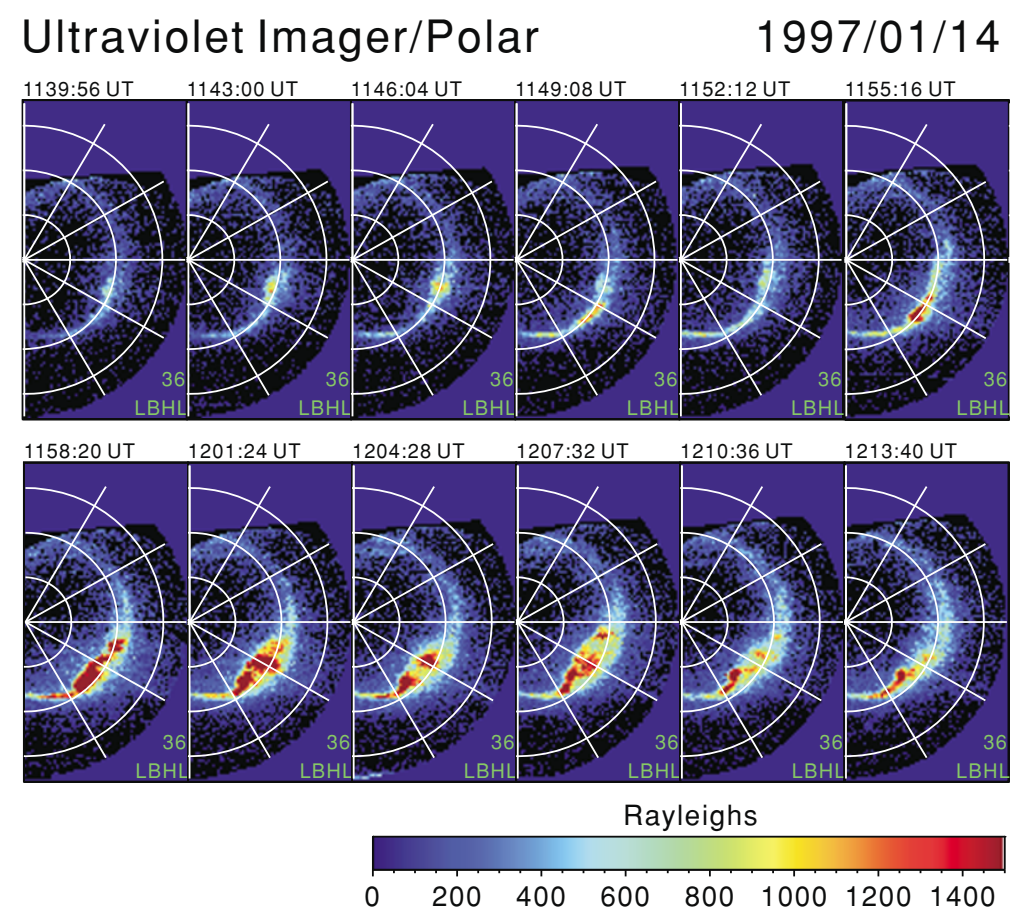

Fig. 1. Polar/UVI data from 1139:56 UT to 1213:40 UT on January 14, 1997 in the MLT-GMLAT plane. White straight lines represent MLTs counterclockwise from 1800 MLT to 0600 MLT through 0000 MLT with a 2 hour increment. The GMLAT semicircles are drawn every $10^{\circ}$ between $60^{\circ}$ and $90^{\circ}$.

located around $\sim \pm 30^{\circ}$ geomagnetic latitude (GMLAT) and separated by $\sim 120^{\circ}$ in geomagnetic longitude (GMLON). We statistically examined geomagnetic field variations in the $\mathrm{Pi} 2$ frequency band observed simultaneously at the both stations. Since Pi2 pulsations are usually observed at substorm onset, we used auroral breakups identified from the Polar auroral images as substorm onsets. We determined MLT of auroral breakups and sorted characteristics of Pi2 pulsations as a function of $\triangle \mathrm{MLT}$, which is the difference in MLT between ground stations and auroral breakup.

The rest of the paper is organized as follows. In Section 2 we describe data set. Section 3 shows an example of Pi2 pulsation observed at both Kakioka and Hermanus on January 14, 1997. In Section 4 statistical results of Pi2 pulsations are displayed. Longitudinal dependence of Pi2 amplitude and phase difference will be revealed. In Section 5 we discuss the statistical results and propose a possible longitudinal structure of the plasmaspheric cavity mode. Section 6 presents conclusions.

\section{Data Set}

\subsection{Geomagnetic field data}

We used geomagnetic field data obtained at the Kakioka (27.2 $2^{\circ}$ GMLAT, 208.5 ${ }^{\circ}$ GMLON) and Hermanus $\left(-33.9^{\circ}\right.$ GMLAT, 82.2 ${ }^{\circ}$ GMLON) magnetic observatories. The Hermanus observatory is located west of the Kakioka observatory by $126.3^{\circ}$ of GMLON or 8.4 hours of MLT.

Study of Pi2 pulsations, in particular, study of their longitudinal characteristics by multiple stations, requires geomagnetic field data recorded at high time-resolution with high accuracy of timekeeping. This requirement can be fulfilled by the Kakioka and Hermanus observatories that provide 1 second geomagnetic field data. At the Kakioka ob- servatory, time is calibrated automatically with overall accuracy of 5 milliseconds by receiving the JJY time signal (10 MHz in the shortwave band) until June 1999 (Oowada et al., 2003). Since June 1999 time calibration has been made by the GPS time signal. At the Hermanus observatory the time calibrations were made by time signals from a national FM radio station with accuracy better than $0.3 \mathrm{sec}$ before 2000, and by the GPS time signal after 2001 .

\subsection{Auroral image data}

We used auroral image data from the ultraviolet imager (UVI) on board the Polar spacecraft, which is documented by Torr et al. (1995). UVI provides auroral luminosity over large areas covering the polar cap, the oval, and sub-auroral zone. Figure 1 shows a sequence of nightside UVI auroral images from 1139:56 UT to $1213: 40$ UT on January 14, 1997 in the MLT-GMLAT plane. In each panel there are seven diverging/converging lines like wheel spokes with each representing an even integer hour from 1800 MLT to 0600 MLT through 0000 MLT, counterclockwisely. The largest semicircle represents $60^{\circ}$ GMLAT and the center of semicircles represents $90^{\circ}$ GMLAT. Auroral brightness is measured at the Lyman-Birge-Hopfield long (LBHL) wavelength ( 160-180 nm). Each UVI image is integrated over a $36 \mathrm{sec}$ interval which is centered at time indicated above individual frame, and is sampled typically at every $184 \mathrm{sec}$ (3.07 $\mathrm{min})$.

We converted the auroral brightness (in Reyleighs) to precipitating electron energy flux (in erg. $\mathrm{cm}^{-2} \cdot \mathrm{s}^{-1}$ ) in the same way as Brittnacher et al. (1997) and Liou et al. (1998) (110 Reyleighs $\approx 1 \mathrm{erg} \cdot \mathrm{cm}^{-2} \cdot \mathrm{s}^{-1}$ ), and then integrated the energy flux over a fan-shaped region of 1 hour MLT by $30^{\circ}$ GMLAT $\left(60^{\circ}-90^{\circ}\right.$ GMLAT) to derive energy dissipation (in gigawatts or GW) at every 1 hour MLT bin. This generates 
12 sets of time series data of auroral energy dissipation, each of which corresponds to the 1 hour MLT bin from 1800-1900 MLT to 0500-0600 MLT. Figure 2 shows an example of calculated energy dissipation for 0500-1400 UT on January 14, 1997 . The time interval of Fig. 1 is indicated by a meshed box. Each of the 12 panels displays time series of auroral power for each 1 hour MLT bin as indicated within the panel. Such a time series of auroral energy dissipation was used to identify the onset time and MLT of auroral breakups. (Arrows indicate onset of auroral breakups. See Section 4.1).

\subsection{Period of investigation}

We used the ground magnetometer data and the Polar/UVI data for the period of December 4, 1996 to March 3, 1997, during which the UVI images of the northern polar region are subject to little effects from the sunlight. Geomagnetically disturbed days with the minimum value of Dst less than $-30 \mathrm{nT}$ were excluded from the analysis, because the geomagnetic field is strongly perturbed in those days and $\mathrm{Pi} 2$ pulsations can be masked by such perturbation. There were 19 days with $\mathrm{Dst}_{\min }<-30 \mathrm{nT}$ in the aforementioned period, thus number of days investigated here is 71 days in total.

\section{Case Study: The January 14, 1997, Event}

Figure 1 shows intermittent auroral activity characterized by two pseudo-breakups around 2230 MLT in the 1143:00 UT frame and around 2130 MLT in the 1149:08 UT frame, and a breakup around 2130 MLT in the 1155:16 UT frame. The breakup is followed by poleward and azimuthal (westward and eastward) expansions of the auroral arc. From the energy dissipation data shown in Fig. 2, we found very small bumps around 1145 UT in the 2100-2200 MLT and 2200-2300 MLT panels; these bumps result from the two pseudo-breakups. After 1150 UT there is a clear enhancement of the energy dissipation with more than $2.5 \mathrm{GW}$. This enhancement started at 2100-2200 MLT and expanded toward earlier and later MLTs.

Figure 3 shows the bandpass-filtered $\mathrm{H}$ component of the geomagnetic field data from Kakioka and Hermanus for 1136-1216 UT on January 14, 1997. Period range of bandpass filter is 30-200 s. At 1200 UT, Kakioka was located at MLT=21.2 hours, being close to MLT where the pseudo-breakups or the breakup were initiated. Hermanus was on the dayside (MLT=12.9 hours) at 1200 UT. Horizontal bars at the bottom of Fig. 3 represent the $36 \mathrm{sec}$ intervals corresponding to each frame in Fig. 1. There are no clear variations both at Kakioka and Hermanus for the first pseudo-breakup (the 1143:00 UT frame), while Pi2 pulsations appeared for the second pseudo-breakup (the 1149:08 frame) with small amplitude. They have similar waveforms and no phase shift. When the auroral breakup occurred (the 1155:16 UT frame and afterwards), both Kakioka and Hermanus observed small-amplitude $\mathrm{Pi} 2$ pulsations that started at 1154:30 UT and continued for $\sim 2$ cycles, as well as clear $\mathrm{Pi} 2$ pulsations starting at $1157 \mathrm{UT}$ with a peak amplitude of $\sim 1.8 \mathrm{nT}$ at Kakioka and $\sim 0.8 \mathrm{nT}$ at Hermanus. It is worthwhile to note that $\mathrm{Pi} 2$ pulsations were observed even on the dayside $(\sim 1300$ MLT). Pi2 frequency was almost the same $(\sim 10 \mathrm{mHz})$. We found no phase shift between the Pi2 pul-

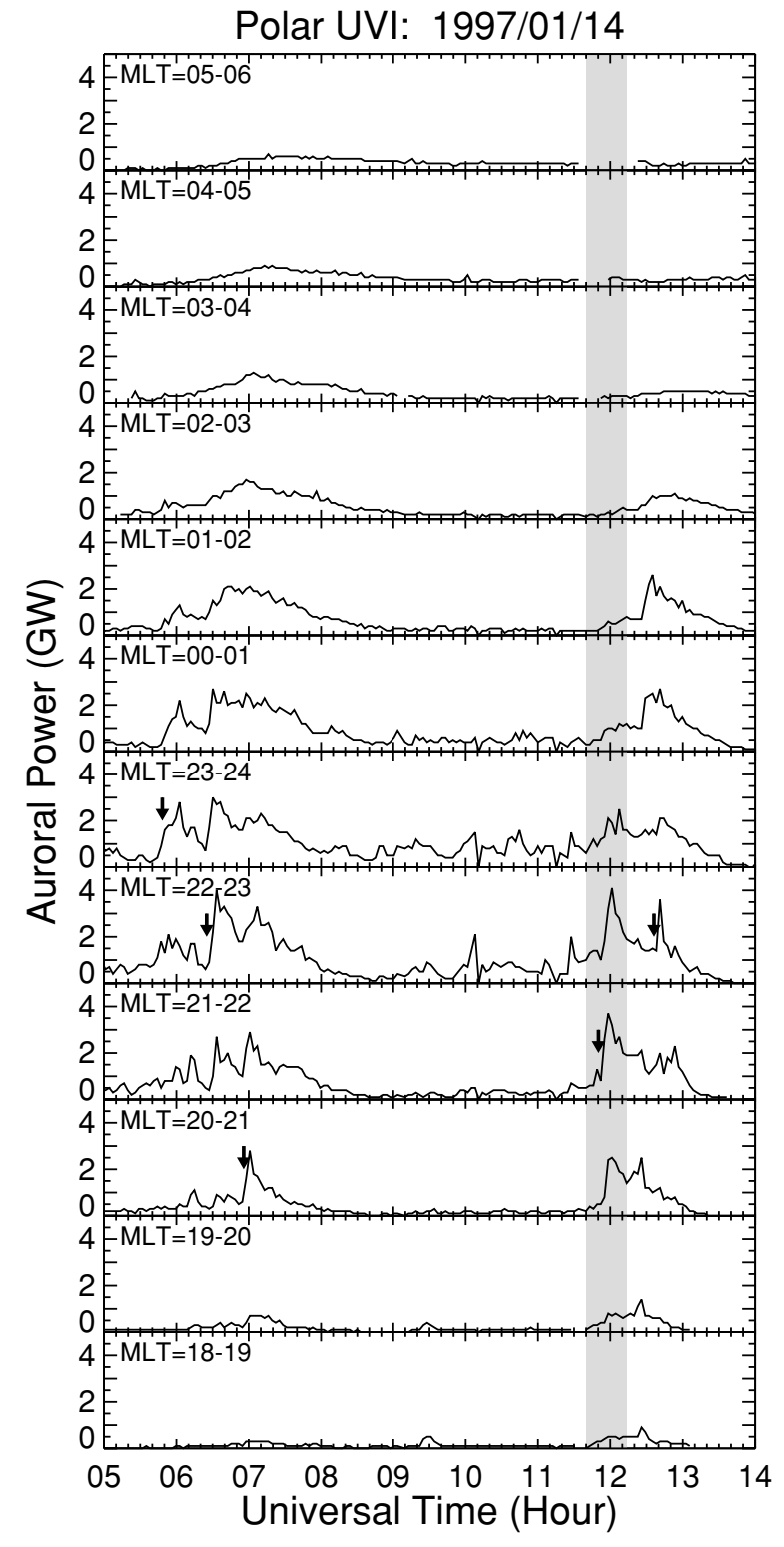

Fig. 2. Auroral power integrated over the 1 hour bin of MLT by $60-90^{\circ}$ bin of GMLAT for 0500-1400 UT on January 14, 1997. Each panel corresponds to 1 hour bin of MLT. A vertical meshed box indicates the time interval shown in Fig. 1. Arrows indicate onset of auroral breakups identified by the selection procedure.

sations at Kakioka and Hermanus, even though these two stations are separated by 8.4 hours of MLT.

\section{Statistical Study \\ 4.1 Selection of auroral breakups}

For a statistical analysis of low-latitude $\mathrm{Pi} 2$ pulsations we detected auroral breakups (i.e., substorms), using the time series data of the auroral energy dissipation, which were derived from the UVI data as explained in Section 2.2. This is because $\mathrm{Pi} 2$ pulsations are excited when auroral breakups occur, as can be seen in Figs. 1 and 3. The following two criteria were used to select possible auroral breakups: (1) energy dissipation increases by more than $1.0 \mathrm{GW}$ within 3.07 min (1 frame); and (2) energy dissipation continues and it increases by more than $2.0 \mathrm{GW}$ within $12.3 \mathrm{~min}(4$ frames). Criterion 1 is required to choose an event with 


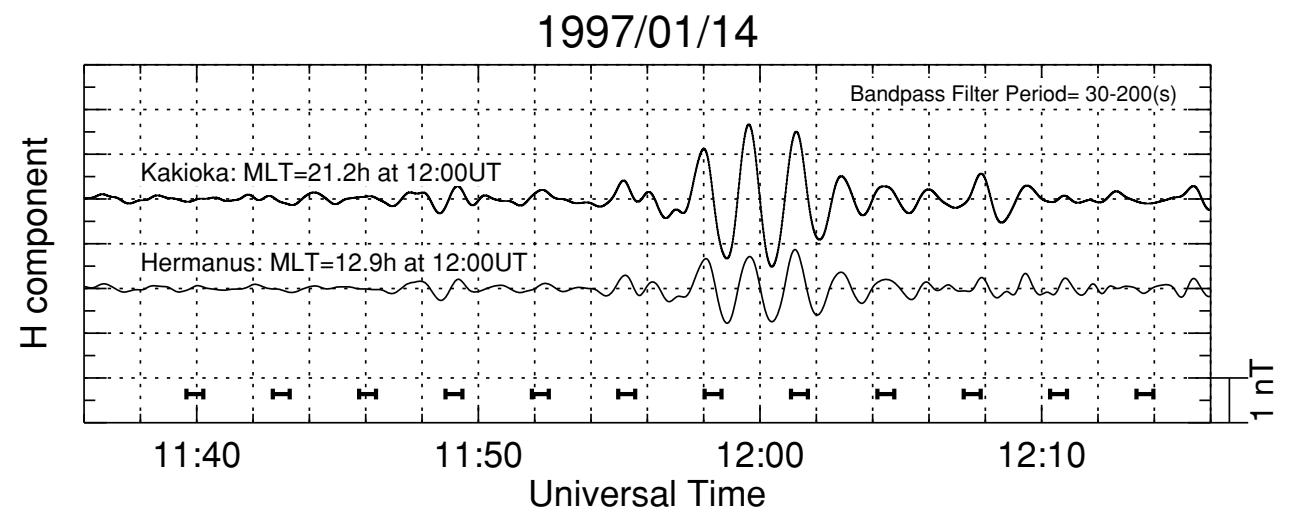

Fig. 3. The bandpass-filterd H component of the geomagnetic field data recorded at Kakioka and Hermanus for 1136-1216 UT on January 14 , 1997. Horizontal bars at the bottom show the $36 \mathrm{sec}$ intervals corresponding to the Polar/UVI images of Fig. 1.

sudden activation of aurora (breakup in literal sense) and criterion 2 is required to exclude pseudo-breakups. These criteria were applied to the 12 sets of energy dissipation data independently. Results of selection were scanned in time sequence for each MLT, and if a given event is separated by more than $30 \mathrm{~min}$ from a previous event, that event was hold for next step. Then we considered events in all MLT bins and checked if more than two events were found in different MLT bins within $30 \mathrm{~min}$. We took the earliest event as a breakup. If more than two events occurred simultaneously, an event with the largest energy dissipation change was taken. The onset time of the breakup is defined as the time of the frame leading to the $1.0 \mathrm{GW}$ increase, and the breakup MLT is defined as the center of the corresponding MLT bin. In Fig. 2, for example, this selection procedure yielded 5 auroral breakups as indicated by vertical arrows, which are consistent with a visual inspection. Note that during the time interval indicated by the meshed box, the breakup is selected but the pseudo-breakups are not. With the selection procedure we obtain 331 breakups during the 71 days.

Figure 4 shows the occurrence frequency of the selected auroral breakups (bar chart, left axis for the scale) and num-

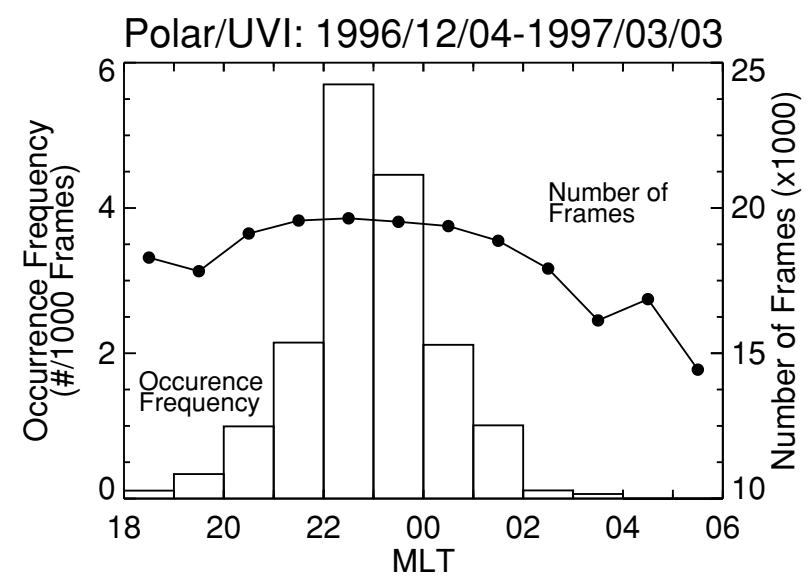

Fig. 4. (Bar chart) MLT dependence of occurrence frequency of the auroral breakups. (Line plot) Number of the Polar/UVI frames used in this study as a function of MLT. ber of the UVI image frames (line plot, right axis for the scale) as a function of MLT. The number of frames on the dawnside is smaller than that near midnight, but it is still enough number for statistics $(\sim 15,000$ frames $)$ to derive the occurrence frequency. We found that occurrence of auroral breakups has a peak at 2200-2300 MLT and decreases monotonically with a MLT distance from there. Most of breakups (about 85\%) are initiated between 2100 MLT and 0100 MLT.

\subsection{Analysis method of $\mathbf{P i 2}$}

We performed spectrum analysis for the $\mathrm{H}$ component of the geomagnetic field data from both Kakioka and Hermanus. The geomagnetic field data were analyzed for a $10 \mathrm{~min}$ interval (i.e., 600 data points) starting from the onset time of auroral breakups. Using the fast Fourier transformation (FFT) method, we calculated power spectra $(p(f)$, where $f$ is the frequency) of the $10 \mathrm{~min}$ segments of Kakioka and Hermanus data for the 331 events. We searched a peak of the power spectrum in the Pi2 frequency range $(6.6-25 \mathrm{mHz})$. The frequency that gives the peak was defined as a dominant frequency $\left(f_{\mathrm{KAK}}\right.$ or $\left.f_{\mathrm{HER}}\right)$. If there are more than one peaks in the $\mathrm{Pi} 2$ frequency range, we took a peak of the highest power. Amplitude of waves $\left(A_{\mathrm{KAK}}\right.$ or $\left.A_{\mathrm{HER}}\right)$ was calculated by $\left(\int_{f_{1}}^{f_{2}} p(f) d f\right)^{1 / 2}$, where $f_{1}$ and $f_{2}$ are $6.6 \mathrm{mHz}$ and $25 \mathrm{mHz}$, respectively. In case of no spectral peak, the dominant frequency and the wave amplitude were not defined. Regarding coherence (coh) and phase difference $(\Delta \Phi)$ between Kakioka and Hermanus, we first chose the reference station which has a smaller $|\triangle \mathrm{MLT}|$, where $\triangle \mathrm{MLT}$ is defined as MLT of a station subtracted by MLT of an auroral breakup. (A positive $\triangle$ MLT means that the station is located geomagnetically eastward of the auroral breakup site.) Then we calculated coherence and phase difference at the dominant frequency of the reference station. Letting $\Phi_{\text {ref }}\left(\Phi_{\text {nonref }}\right)$ be a phase of wave at the reference (non-reference) station, we defined $\Delta \Phi$ as $\Phi_{\text {nonref }}-\Phi_{\text {ref. }}$ A positive $\Delta \Phi$ represents that the wave at the reference station lags behind that at the non-reference station.

Figure 5 shows an example of the above analysis for the Pi2 event presented in Fig. 3 (the January 14, 1997, event). Data period analyzed is 1152:12-1202:12 UT. Top panel demonstrates power spectra for Kakioka (thick line) and 

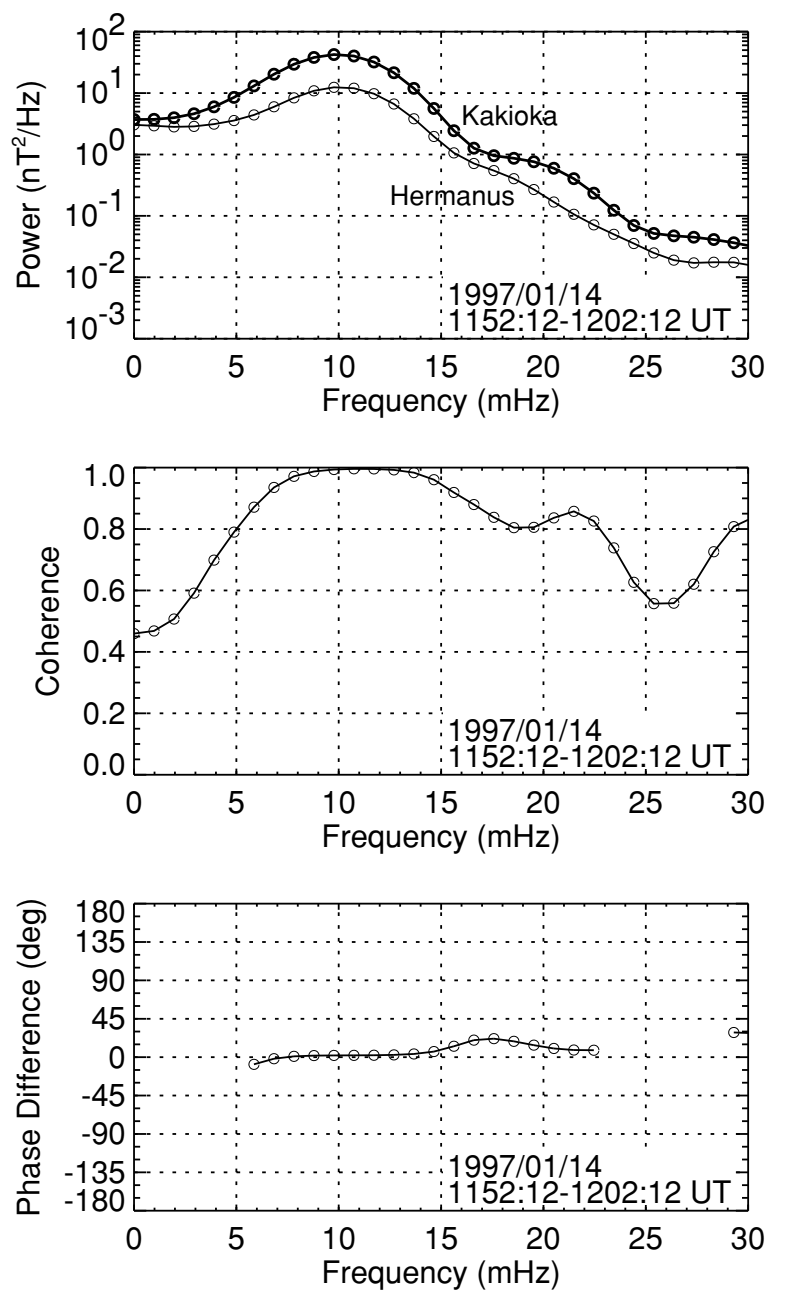

Fig. 5. Example of the spectrum analysis of the Pi2 event shown in Fig. 3. (top) power spectra of Kakioka and Hermanus. (middle) coherence between Kakioka and Hermanus. (bottom) Phase difference which is defined as phase at Hermanus minus phase at Kakioka. Only shown at frequencies with coherence larger than or equal to 0.8 .

Hermanus (thin line). Middle and bottom panels are for coherence and phase difference, respectively. Phase differences are only shown at frequencies with $\operatorname{coh} \geq 0.8$. The power spectra give dominant frequency at $9.8 \mathrm{mHz}$ for both stations with $A_{\mathrm{KAK}}=0.51 \mathrm{nT}$ and $A_{\mathrm{HER}}=0.28 \mathrm{nT}$. Since Kakioka was closer to the auroral breakup region in MLT than Hermanus, we chose Kakioka as the reference station and found $c o h=0.99$ and $\Delta \Phi=\Phi_{\mathrm{HER}}-\Phi_{\mathrm{KAK}}=2.1^{\circ}$ at $f_{\mathrm{KAK}}=9.8 \mathrm{mHz}$. These values agree to the visual inspection of Fig. 3.

\subsection{Wave amplitude}

Figures 6(a) and 6(b) display $\triangle$ MLT dependence of $A_{\mathrm{KAK}}$ and $A_{\mathrm{HER}}$, respectively. Open circles indicate wave amplitude of individual events and filled circles indicate the mean values of wave amplitude in 2 hour $\triangle$ MLT bins. Error bars are standard errors. From Fig. 6(a) we found that $A_{\mathrm{KAK}}$ has peak at $\triangle \mathrm{MLT}=-2$ to 4 hours and decreases when $|\triangle \mathrm{MLT}|$ becomes large. The distribution of $A_{\mathrm{KAK}}$ is almost symmetric against $\triangle \mathrm{MLT}=0-2$ hours. Figure $6(\mathrm{~b})$ shows a peak of $A_{\mathrm{HER}}$ at $\Delta \mathrm{MLT}=-2$ to 0 hours. However, the distribution of $A_{\mathrm{HER}}$ is not symmetric in $\triangle \mathrm{MLT}$; that is, $A_{\mathrm{HER}}$ around $\triangle \mathrm{MLT}=-10$ to -4 hours is larger than $A_{\mathrm{HER}}$ around $\triangle \mathrm{MLT}=4$ to 10 hours. Since the $\Delta \mathrm{MLT}$ range of -10 to -4 hours roughly corresponds to the afternoon sector and Hermanus is located at higher GMLAT than Kakioka, we think that the asymmetry of $A_{\mathrm{HER}}$ is caused by Pc3-4 waves which occur predominantly in the afternoon sector and have larger amplitude at higher latitude with a local maximum at 40-50 GMLAT (e.g., Yumoto et al., 1992; Ziesolleck et al., 1993; Matsuoka et al., 1997). We conclude from Fig. 6 that wave power of $\mathrm{Pi} 2$ pulsations is highest near the auroral breakup meridian $(|\Delta \mathrm{MLT}| \leq 4$ hours) and decreases as a ground station is separated longitudinally from the breakup region.

\subsection{Coherence}

In the January 14, 1997, event, the waveform of dayside Pi2 pulsation was very similar to that of nightside Pi2 pulsation as shown in Figs. 3 and $5(\operatorname{coh}=0.99)$. Thus we statistically investigated coherence of variations at the $\mathrm{Pi} 2$ frequency band between Kakioka and Hermanus. Only events in which $\triangle$ MLT of the reference station is -4 to 4 hours were used, because we intend to compare Pi2 waveforms between meridians close to and far from the auroral breakup region. Figure 7(a) represents a result when $\triangle$ MLT of Kakioka was -4 to 4 hours; that is, Kakioka was the reference station and close to the auroral breakup meridian. Hermanus was approximately located from noon
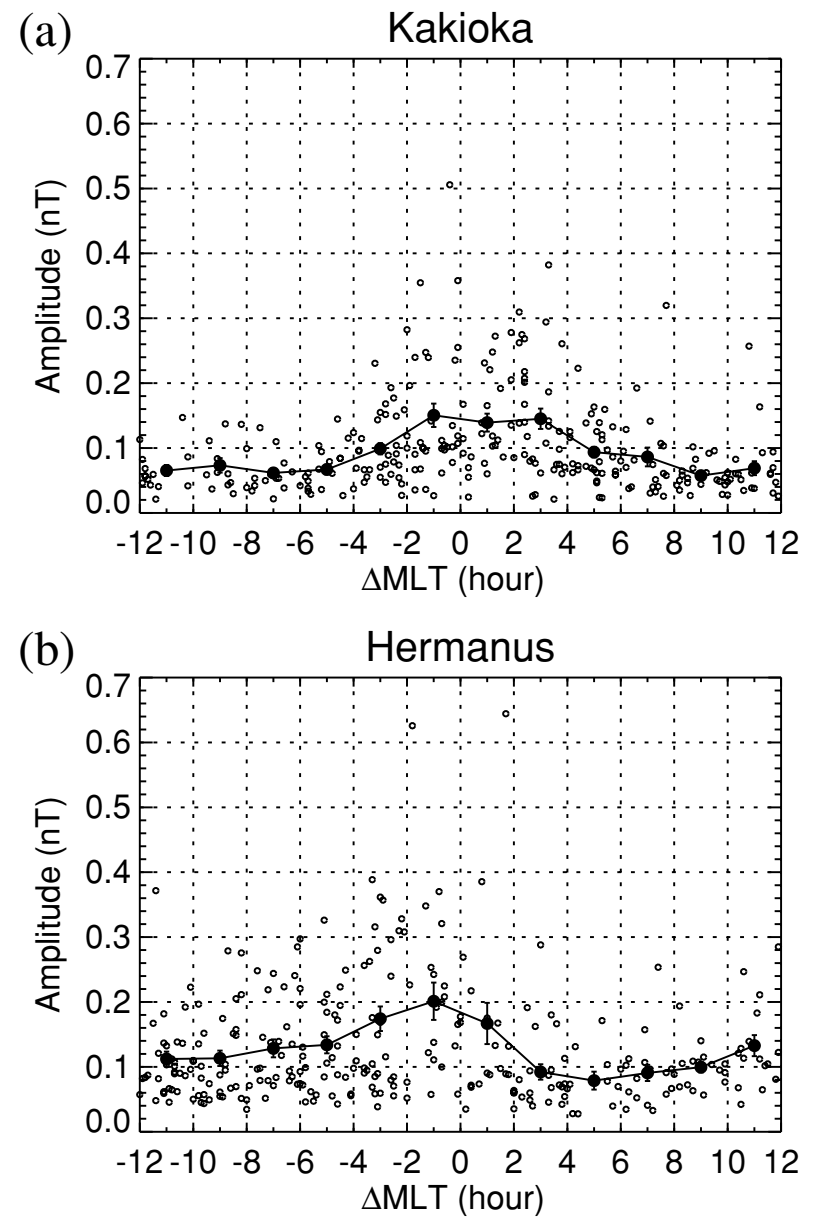

Fig. 6. (a) $\triangle$ MLT dependence of Pi2 amplitude at Kakioka. (b) The same as Fig. 6(b) except for Hermanus. 
(a)

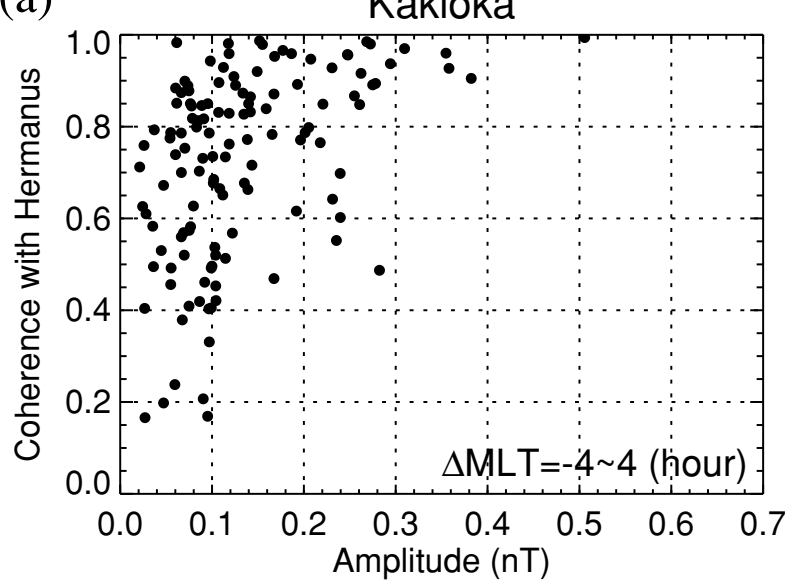

(b)

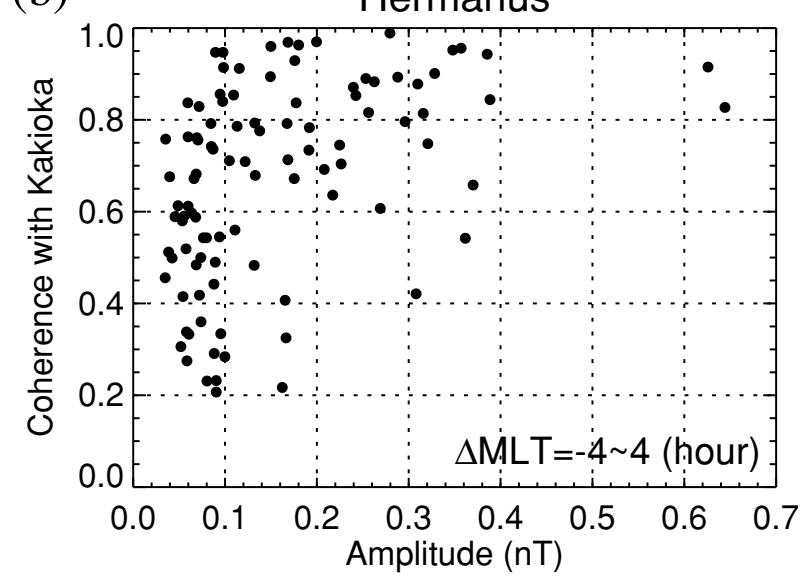

Fig. 7. (a) Coherence of Pi2 pulsations between Kakioka and Hermanus when Kakioka is the reference station. (b) The same as Fig. 7(a) except for Hermanus as the reference station.

to the evening sector. Coherence was shown as a function of $A_{\mathrm{KAK}}$. Individual events were indicated with filled circles. We found that the coherence is generally proportional to $A_{\mathrm{KAK}}$. When $A_{\mathrm{KAK}} \geq 0.2 \mathrm{nT}$, occurrence rate of events with $\operatorname{coh} \geq 0.8$ is $69 \%(18 / 26)$, while the rate decreases to $26 \%(15 / 57)$ when $A_{\mathrm{KAK}}<0.1 \mathrm{nT}$. Figure $7(\mathrm{~b})$ is the same as Fig. 7(a) except for Hermanus as the reference station. In this case, Hermanus was near the breakup meridian and Kakioka was located from the morning sector to noon. The coherence generally becomes high as $A_{\mathrm{HER}}$ becomes large. When $A_{\mathrm{HER}} \geq 0.2 \mathrm{nT}$, occurrence rate of events with $c o h \geq 0.8$ was $62 \%(16 / 26)$ and it becomes $15 \%(7 / 48)$ when $A_{\mathrm{HER}}<0.1 \mathrm{nT}$. These results mean that if wave amplitude of $\mathrm{Pi} 2$ pulsations near auroral breakups (on the nightside) is large enough, $\mathrm{Pi} 2$ pulsations with similar waveforms can be seen even on the dayside.

\subsection{Phase difference}

The case study of the January 14,1997 , event has shown $\Delta \Phi \sim 0^{\circ}$, indicating in-phase oscillation of Pi2 pulsations at Kakioka and Hermanus. Here we examine $\Delta \Phi$ statistically, using events in which $\triangle$ MLT of the reference station is -4 to 4 hours and coherence is larger than or equal to 0.95 . Number of events satisfying these criteria was 14 for Kakioka as the reference station, and 6 for Hermanus. Fig- ure 8 demonstrates $\Delta \Phi$ as a function of $\triangle$ MLT for the 14 events (filled circle) and the 6 events (open circle). From Fig. 8 we noticed that $\Delta \Phi$ is almost independent to $\Delta$ MLT. It seems that events can be classified into two groups according to the value of $\Delta \Phi$; one is a group with $\Delta \Phi \sim 0^{\circ}$ and the other is a group with $|\Delta \Phi|=30-60^{\circ}$. In the first group there were 13 events having $|\Delta \Phi|<22.5^{\circ}$. Average of $\Delta \Phi\left(\Delta \Phi_{\text {ave }}\right)$ in this group was calculated as $3.3^{\circ} \pm 2.2^{\circ}$ (uncertainty is the standard error). In the second group we found 7 events and average value of $|\Delta \Phi|$ of $50.6^{\circ} \pm 2.8^{\circ}$. Figure 9 shows an example of events in the second group with $\Delta \Phi=\Phi_{\mathrm{HER}}-\Phi_{\mathrm{KAK}}=46.8^{\circ}$ and $f_{\mathrm{KAK}}=f_{\mathrm{HER}}=9.8 \mathrm{mHz}$ observed on December 7, 1996. Oscillation started almost simultaneously, but time differences between Kakioka and Hermanus appeared for the second to fourth peaks and they were almost constant at $\sim 10 \mathrm{~s}$.

The above results indicate that more than half of $\mathrm{Pi} 2$ pulsations observed at Kakioka and Hermanus oscillate in phase $\left(\Delta \Phi \sim 0^{\circ}\right)$, as the January 14,1997 , event. However, some of Pi2 pulsations have apparent phase difference $\left(|\Delta \Phi|=30-60^{\circ}\right)$ between Kakioka and Hermanus, as the December 7, 1996, event.

One may argue that $\Delta \Phi$ includes not only longitudinal effects but also latitudinal effects, since Kakioka and Hermanus were in different hemispheres and their absolute GMLAT are slightly different by $6.7^{\circ}$. However, previous studies employing latitudinal array of geomagnetic stations showed that $\mathrm{Pi} 2$ pulsations have no phase shift in the $\mathrm{H}$ component at $|\mathrm{GMLAT}|<55^{\circ}$ (Yumoto et al., 1994; Osaki et al., 1996; Li et al., 1998). Kurchashov et al. (1987) studied latitudinal phase differences caused by the coupling of cavity mode and Alfvén waves and found that it is very small (less than $\sim 5^{\circ}$ ). Therefore we suppose that latitudinal effects on $\Delta \Phi$ are negligible.

\section{Discussion}

\subsection{Longitudinal structure of plasmaspheric cavity mode}

We have statistically analyzed low-latitude Pi2 pulsations observed at Kakioka and Hermanus to find longitudinal structure of the plasmaspheric cavity mode. Results

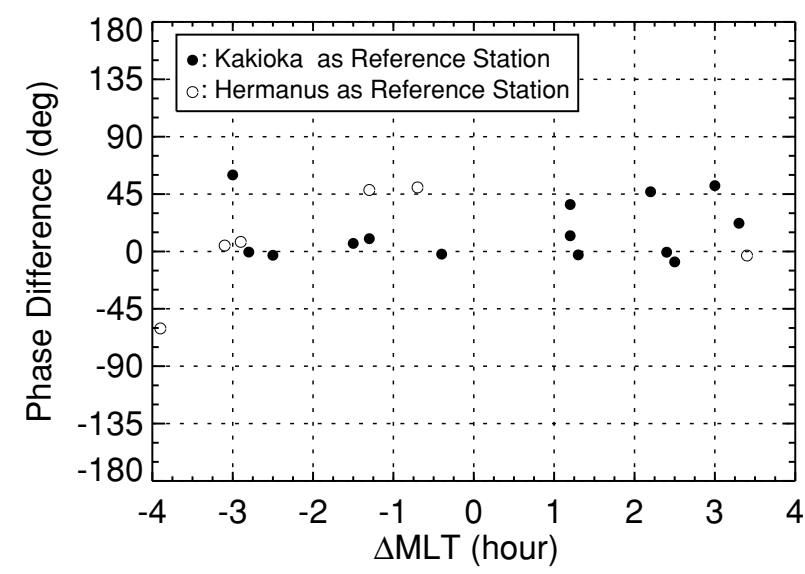

Fig. 8. Phase difference of Pi2 pulsations between Kakioka and Hermanus as a function of $\triangle \mathrm{MLT}$ of the reference station. Filled and open circles represent Kakioka and Hermanus as the reference station, respectively. 


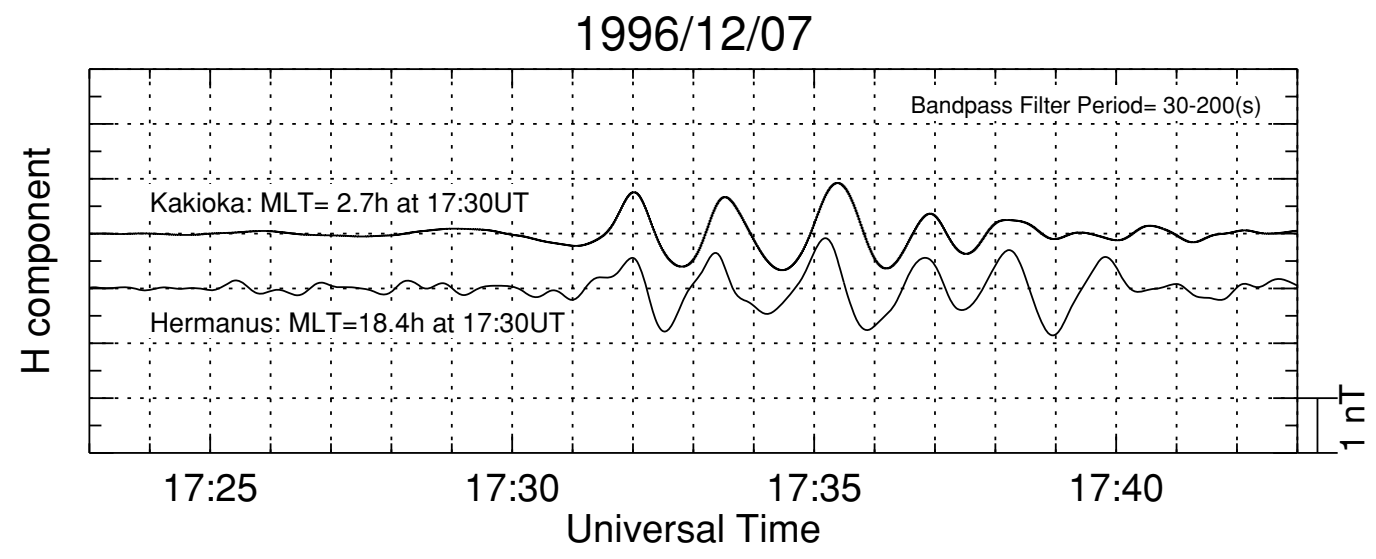

Fig. 9. The same as Fig. 3 except for the period of 1723-1743 UT on December 7, 1996.

are summarized as follows: (1) $\mathrm{Pi} 2$ amplitude has a peak around auroral breakup region $(|\Delta \mathrm{MLT}| \leq 4$ hours); (2) $\mathrm{Pi} 2$ pulsations can be seen on both the nightside and the dayside by similar waveforms if the nightside $\mathrm{Pi} 2$ pulsation has large amplitude; (3) dayside $\mathrm{Pi} 2$ pulsations generally have the in-phase relation with nightside $\mathrm{Pi} 2$ pulsations $\left(\Delta \Phi_{\mathrm{ave}}=3.3^{\circ}\right.$ ), except for some examples having phase differences $\left(|\Delta \Phi|_{\mathrm{ave}}=50.6^{\circ}\right)$; and (4) there is no clear $\Delta$ MLT dependence in $\Delta \Phi$.

Longitudinal wave number of pulsation $(m)$ can be computed by $m=(24 / \Delta \mathrm{MLT}) \times\left(\Delta \Phi / 360^{\circ}\right)$ (e.g., Yumoto et al., 1986). From result 3 we argue that $m$-number of lowlatitude $\mathrm{Pi} 2$ pulsations is 0 in general, because $\Delta \Phi_{\mathrm{ave}}=3.3^{\circ}$ and $\triangle \mathrm{MLT}=8.4$ hours plugged in the above equation give $m=0.026 \approx 0$. This is consistent with the result of Kitamura et al. (1988) and Nosé et al. (2003). From $m \approx 0$ and result 4 , we think that these $\mathrm{Pi} 2$ pulsations are likely to be caused by the global plasmaspheric cavity mode. Figure 10 illustrates oscillation of the global cavity mode in the equatorial plane inferred from the observations. The plasmapause is depicted as an outermost circle for simplicity. A solid green circle shows the positions of the field lines in the middle of the plasmasphere before oscillation starts. Fast mode Alfvén waves are emitted at substorm onset, and they are trapped in the plasmasphere, causing radial oscillation of field lines in the plasmasphere. Since $m \approx 0$, the field lines shown by the solid green circle are though to change their positions as indicated by dotted green circles at maximum displacement. The amplitude is the largest around the auroral breakup MLT and becomes small when $\triangle$ MLT becomes large, as suggested by result 1 . If the amplitude of oscillation on the nightside is large enough, the oscillation on the dayside is expected to have large amplitude accordingly and can be observed as dayside Pi2 pulsations with similar waveforms on the ground. When oscillation on the nightside is small in amplitude, the dayside oscillation becomes small and is easily masked by dayside disturbances such as Pc3-4 pulsations, resulting in low coherence. This explains result 2 and Fig. 7.

On the other hand, the events having $|\Delta \Phi|=30^{\circ}-60^{\circ}$ yield $|m|=0.24-0.48$, which is a non-integer but rather close to 0 . It should be noted that 6 out of the 7 events have positive $\Delta \Phi$, indicating that $\mathrm{Pi} 2$ pulsations observed at the reference station lags behind that at the non-reference station, as shown in Fig. 9. It is difficult to explain the positive $\Delta \Phi$ by propagation of waves from the auroral breakup MLT to the dayside, because the reference station is closer to the auroral breakup MLT than the non-reference station is. We surmize that even in these events the plasmaspheric cavity mode was established primarily in the similar way as Fig. 10 but some processes which cause the phase delay in the reference station were effective. Ionospheric conductivity may be one candidate. Takahashi et al. (1999b) reported a Pi2 pulsation having a small phase difference $\left(17^{\circ}\right)$ in the north-south component of the geomagnetic field oscillation between the ground and a satellite at altitude of $\sim 580 \mathrm{~km}$. Sutcliffe and Lühr (2003) found a Pi2 pulsation in which the $\mathrm{H}$ component oscillated with a slight delay against the compressional component observed by the CHAMP satellite at $\sim 450 \mathrm{~km}$ altitude. If the ionospheric conductivity controls a phase change of the geomagnetic field oscillation during transmission of waves from the plasmasphere to the ground, difference of the conductivity between the dayside and the nightside is a possible cause. The plasmapause

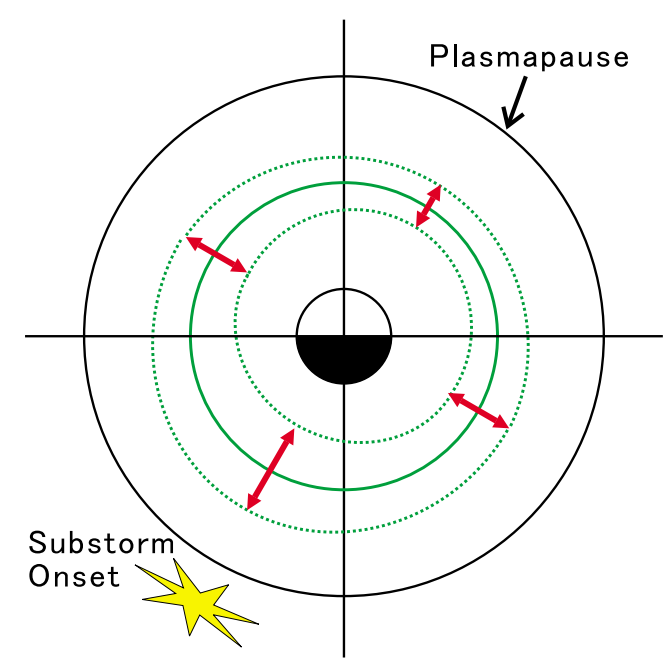

Fig. 10. Schematic figure of longitudinal structure of the plasmaspheric cavity mode to explain the observation. The outermost circle represents the plasmasphere. A solid line and dotted green lines show the positions of the field lines at rest and at maximum displacement, respectively. 


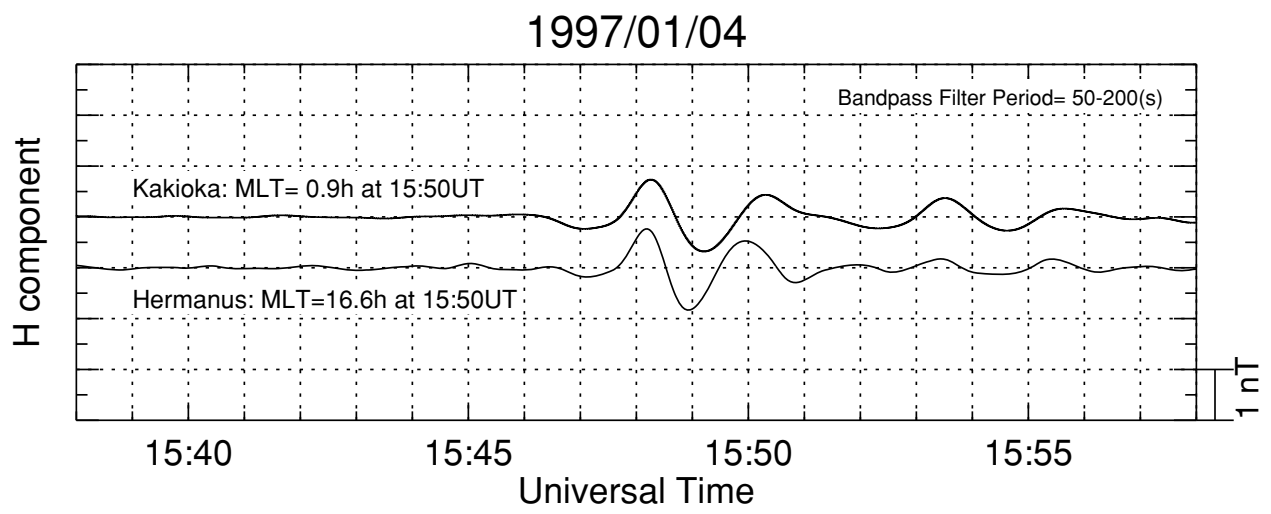

Fig. 11. The same as Fig. 3 except for the period of 1538-1558 UT on January 4, 1997.

may be considered as another candidate. The plasmapause is thought to be a non-perfect reflector for the fast mode waves, leading to leakage of the wave energy to the plasma trough (Fujita and Glassmeier, 1995; Lee and Lysak, 1999; Fujita et al., 2000, 2002). If the condition of the plasmapause is different between the nightside and the dayside, the reflection (and transmission) rate of the fast mode waves at the plasmapause is expected to be different and may give rise to the phase shift between the nightside and the dayside.

It might be possible to attribute the positive $\Delta \Phi$ to different excitation mechanism of Pi2 pulsations on the dayside. Sastry et al. (1983), Shinohara et al. (1997), and Yumoto et al. (2001) suggested that dayside $\mathrm{Pi} 2$ pulations are caused by instantaneous penetration of oscillating electric field from the nightside polar region to the dayside ionosphere. If the electric field penetration takes place faster than the time scale for establishing the cavity mode, we can expect positive $\Delta \Phi$, although we need a theory to explain why the oscillating electric field in the polar region and the cavity mode have identical frequencies. Further investigation using the geomagnetic field data from denser longitudinal magnetometer chain and/or the ionospheric electric field data from radars is needed to interpret the events having small positive values of $\Delta \Phi$.

\subsection{Pi2 pulsations with different periods between} Kakioka and Hermanus

Visually scanning the data plots, we noticed that the periods of some $\mathrm{Pi} 2$ pulsations are different between Kakioka and Hermanus, while most of $\mathrm{Pi} 2$ pulsations showed the same dominant period like the January 14, 1997, event (Fig. 3) and the December 7, 1996, event (Fig. 9). Figure 11 displays an example of such $\mathrm{Pi} 2$ pulsations occurred on January 4, 1997. It is clearly shown that the period of the $\mathrm{Pi} 2$ pulsation observed at Hemanus $(T \sim 100 \mathrm{~s})$ was shorter than that at Kakioka $(T \sim 120 \mathrm{~s})$.

Kosaka et al. (2002) and Han et al. (2003) showed that the dominant frequency of mid- and low-latitude Pi2 pulsations depends on LT. Using geomagnetic field data from Crozet ( $-51.5^{\circ}$ GMLAT), Port Aux Francais (-57.2 ${ }^{\circ} \mathrm{GM}-$ LAT), and Kakioka, Kosaka et al. (2002) revealed that frequency of nightside $\mathrm{Pi} 2$ pulsations becomes higher as a ground station moves from the duskside to the dawnside. From simultaneous observations with 5 low-latitude
( $L=1.2-1.6$ ) stations in Japan and China, Han et al. (2003) found the similar LT dependence of frequency of nightside Pi2 pulsations. Fujita and Itonaga (2003) reproduced this LT dependence of Pi 2 frequency by a numerical simulation of the plasmaspheric cavity resonance (or the plasmaspheric virtual resonance), where longitudinal non-uniformity was introduced in the plasmaspheric Alfvén velocity. They considered the plasmasphere with the largest Alfvén velocity on the dawnside and the smallest Alfvén velocity on the duskside, and found that two kinds of the eigenmodes are established with higher frequency on the dawnside and lower frequency on the duskside. Thus we suggest that the $\mathrm{Pi} 2$ pulsations shown in Fig. 11 can be also excited by the plasmaspheric cavity mode in the longitudinally nonuniform plasmasphere.

\section{Conclusions}

Pi2 pulsations observed at longitudinally separated lowlatitude stations, Kakioka (27.2 ${ }^{\circ}$ GMLAT) and Hermanus $\left(-33.9^{\circ}\right.$ GMLAT), have been analyzed statistically to infer the longitudinal structure of the plasmaspheric cavity mode. We first selected 331 auroral breakups from the Polar/UVI data for the period of December 4, 1996 to March 3, 1997. Then the geomagnetic field data from Kakioka and Hermanus were examined for a 10 min segment after the auroral breakups. We statistically examined amplitude, coherence, and phase difference of oscillations in the $\mathrm{Pi} 2$ frequency band. The main results obtained in this study are summarized as follows.

1. Amplitude of pulsations peaks around the auroral breakup $(|\Delta \mathrm{MLT}| \leq 4$ hours) and decreases away from the breakup. (Fig. 6)

2. Large amplitude nightside Pi2 pulsations are accompanied by dayside Pi2 pulsations with similar waveforms. (Fig. 7)

3. When Pi2 pulsations were seen on both the nightside and the dayside, they are generally in phase $\left(\Delta \Phi_{\text {ave }}=3.3^{\circ}\right)$, except in some events apparent phase differences were observed $\left(|\Delta \Phi|_{\text {ave }}=50.6^{\circ}\right)$. (Fig. 8)

4. There was no clear $\Delta$ MLT dependence in $\Delta \Phi$. (Fig. 8)

From these observational results we conclude that the plasmaspheric cavity mode is excited globally (i.e., $m \approx 0$ ) in general. Oscillation of the cavity mode is expected to be the largest around the auroral breakup MLT and small on 
the dayside. A schematic figure for the plasmaspheric cavity mode deduced from the observations is shown in Fig. 10. Regarding some Pi2 events showing phase difference, we suppose that the global plasmaspheric cavity mode is established with some mechanisms which produce the phase difference between the nightside and the dayside.

From visual inspection of data plots we found some Pi2 pulsations having different period between Kakioka and Hermanus such as events shown in Fig. 11. We argue that these Pi2 pulsations are also caused by the cavity mode in the longitudinally non-uniform plasmasphere.

Acknowledgments. We thank K. Takahashi, A. Yoshida, Y. Yamada, T. Koide, T. Tokumoto, T. Iyemori, and D. Han for their helpful comments. The geomagnetic field data from Kakioka were provided by Kakioka Magnetic Observatory. This work was supported by the Sumitomo Foundation (grant 030677) and the Ministry of Education, Science, Sports and Culture, Grant-in-Aid for Young Scientists (B). The work of K. Liou is supported by ASFOR through NSF Space Weather grant ATM 0318606.

\section{References}

Brittnacher, M., R. Elsen, G. Parks, L. Chen, G. Germany, and J. Spann, A dayside auroral energy deposition case study using Polar Ultraviolet Imager, Geophys. Res. Lett., 24, 991-994, 1997.

Fujita, S. and K.-H. Glassmeier, Magnetospheric cavity resonance oscillations with energy flow across the magnetopause, J. Geomagn. Geoelectr., 47, 1277-1292, 1995.

Fujita, S. and M. Itonaga, A plasmaspheric cavity resonance in a longitudinally non-uniform plasmasphere, Earth Planets Space, 55, 219-222, 2003.

Fujita, S., M. Itonaga, and H. Nakata, Relationship between the Pi2 pulsations and the localized impulsive current associated with the current disruption in the magnetosphere, Earth Planets Space, 52, 267-281, 2000.

Fujita, S., H. Nakata, M. Itonaga, A. Yoshikawa, and T. Mizuta, A numerical simulation of the Pi2 pulsations associated with the substorm current wedge, J. Geophys. Res., 107(A3), DOI 10.1029/2001JA900137, 2002.

Han, D., T. Iyemori, Y. Gao, Y. Sano, F. Yang, W. Li, and M. Nosé, Local time dependence of the frequency of $\mathrm{Pi} 2$ waves simultaneously observed at 5 low-latitude stations, Earth Planets Space, 55, 601-612, 2003.

Han, D., T. Iyemori, M. Nosé, H. McCreadie, Y. Gao, F. Yang, S. Yamashita, and P. Stauning, A comparative analysis of low-latitude Pi2 pulsations observed by Ørsted and ground stations, J. Geophys. Res., 109, A10209, doi:10.1029/2004JA010576, 2004.

Keiling, A., J. R. Wygant, C. Cattell, K.-H. Kim, C. T. Russell, D. K. Milling, M. Temerin, F. S. Mozer, and C. A. Kletzing, Pi2 pulsations observed with the Polar satellite and ground stations: Coupling of trapped and propagating fast mode waves to a midlatitude field line resonance, J. Geophys. Res., 106, 25,891-25,904, 2001.

Kim, K.-H., K. Takahashi, D.-H. Lee, N. Lin, and C. A. Cattell, A comparison of $\mathrm{Pi} 2$ pulsations in the inner magnetosphere and magnetic pulsations at geosynchronous orbit, J. Geophys. Res., 106, 18,865-18,872, 2001.

Kitamura, T., O. Saka, M. Shimoizumi, H. Tachihara, T. Oguti, T. Araki, N. Sato, M. Ishitsuka, O. Veliz, and J. B. Nyobe, Global mode of Pi2 waves in the equatorial region: Difference of $\mathrm{Pi} 2$ mode between high and equatorial latitudes, J. Geomagn. Geoelectr., 40, 621-634, 1988.

Kosaka, K., T. Iyemori, M. Nosé, M. Bitterly, and J. Bitterly, Local time dependence of the dominant frequency of $\mathrm{Pi} 2$ pulsations at mid- and low-latitudes, Earth Planets Space, 54, 771-781, 2002.

Kurchashov, Y. P., Y. S. Nikomarov, V. A. Pilipenko, and A. Best, Field line resonance effects in local meridional structure of mid-latitude geomagnetic pulsations, Ann. Geophys., 5A, 147-154, 1987.

Lee, D.-H. and R. L. Lysak, MHD waves in a three-dimensional dipolar magnetic field: A search for Pi2 pulsations, J. Geophys. Res., 104, 28,691-28,699, 1999.

Li, Y., B. J. Fraser, F. W. Menk, D. J. Webster, and K. Yumoto, Properties and sources of low and very low latitude Pi2 pulsations, J. Geophys. Res., 103, 2343-2358, 1998.

Lin, C. C. and L. J. Cahill, Jr., Pi2 pulsations in the magnetosphere, Planet. Space. Sci., 23, 693-711, 1975.

Liou, K., P. T. Newell, C. -I. Meng, M. Brittnacher, and G. Parks, Char- acteristics of the solar wind controlled auroral emissions, J. Geophys. Res., 103, 17,543-17,557, 1998.

Liou, K., C. -I. Meng, P. T. Newell, K. Takahashi, S. -I. Ohtani, A. T. Y. Lui, M. Brittnacher, and G. Parks, Evaluation of low-latitude Pi2 pulsations as indicators of substorm onset using Polar ultraviolet imagery, $J$. Geophys. Res., 105, 2495-2505, 2000.

Matsuoka, H., K. Takahashi, S. Kokubun, K. Yumoto, T. Yamamoto, S. I. Solovyev, and E. F. Vershinin, Phase and amplitude structure of Pc3 magnetic pulsations as determined from multipoint observations, J. Geophys. Res., 102, 2391-2403, 1997.

Nosé, M., K. Takahashi, T. Uozumi, K. Yumoto, Y. Miyoshi, A. Morioka, D. K. Milling, P. R. Sutcliffe, H. Matsumoto, T. Goka, and H. Nakata, Multipoint observations of a $\mathrm{Pi} 2$ pulsation on morningside: The 20 September 1995 event, J. Geophys. Res., 108(A5), 1219, doi:10.1029/2002JA009747, 2003.

Oowada, T., T. Tokumoto, Y. Yamada, M. Ozima, N. Kumasaka, M. Yokoyama, M. Sugawara, K. Koike, and Y. Shimizu, Technical Report of the Kakioka Magnetic Observatory, Supplement, 1, pp. 1-14, 2003.

Osaki, H., K. Yumoto, K. Fukao, K. Shiokawa, F. W. Menk, B. J. Fraser, and the $210^{\circ} \mathrm{MM}$ Magnetic Observation Group, Characteristics of lowlatitude $\mathrm{Pi} 2$ pulsations along the $210^{\circ}$ magnetic meridian, J. Geomagn. Geoelectr., 48, 1421-1430, 1996.

Sakurai, T. and R. L. McPherron, Satellite observations of Pi2 activity at synchronous orbit, J. Geophys. Res., 88, 7015-7027, 1983.

Sastry, T. S., Y. S. Sarma, S. V. Sarma, and P. V. S. Narayan, Day-time Pi pulsations at equatorial latitudes, J. Atmos. Terr. Phys., 45, 733-741, 1983.

Shinohara, M., K. Yumoto, A. Yoshikawa, O. Saka, S. I. Solovyev, E. F. Vershinin, N. B. Trivedi, J. M. Da Costa, and The $210^{\circ}$ MM Magnetic Observation Group, Wave characteristics of daytime and nighttime Pi2 pulsations at the equatorial and low latitudes, Geophys. Res. Lett., 24, 2279-2282, 1997.

Sutcliffe, P. R. and H. Lühr, A comparison of Pi2 pulsations observed by CHAMP in low Earth orbit and on the ground at low latitudes, Geophys. Res. Lett., 30(21), 2105, doi:10.1029/2003GL018270, 2003.

Takahashi, K., S. Ohtani, and B. J. Anderson, Statistical analysis of Pi2 pulsations observed by the AMPTE CCE spacecraft in the inner magnetosphere, J. Geophys. Res., 100, 21,929-21,941, 1995.

Takahashi, K., W. J. Hughes, R. R. Anderson, and S. I. Solovyev, CRRES satellite observations associated with low-latitude Pi2 pulsations, J. Geophys. Res., 104, 17,431-17,440, 1999a.

Takahashi, K., B. J. Anderson, and K. Yumoto, Upper Atmosphere Research Satellite observation of a Pi2 pulsation, J. Geophys. Res., 104, 25,035-25,045, 1999b.

Takahashi, K., S. Ohtani, W. J. Hughes, and R. R. Anderson, CRRES observation of $\mathrm{Pi} 2$ pulsations: Wave mode inside and outside the plasmasphere, J. Geophys. Res., 106, 15,567-15,581, 2001.

Takahashi, K., D.-H. Lee, M. Nosé, R. R. Anderson, and W. J. Hughes, CRRES electric field study of the radial mode structure of Pi2 pulsations, J. Geophys. Res., 108(A5), 1210, doi:10.1029/2002JA009761, 2003a.

Takahashi, K., R. R. Anderson, and W. J. Hughes, Pi2 pulsations with second harmonic: CRRES observations in the plasmasphere, J. Geophys. Res., 108(A6), 1242, doi:10.1029/2003JA009847, 2003 b.

Torr, M. R., et al., A far ultraviolet imager for the international solarterrestrial physics mission, Space Sci. Rev., 71, 329-380, 1995.

Yumoto, K., Generation and propagation mechanisms of low-latitude magnetic pulsations, A review, J. Geophys., 60, 79-105, 1986.

Yumoto, K., Y. Tanaka, T. Oguti, K. Shiokawa, Y. Yoshimura, A. Isono, B. J. Fraser, F. W. Menk, J. W. Lynn, M. Seto, and $210^{\circ}$ MM Magnetic observation Group, Globally coordinated magnetic observations along $210^{\circ}$ Magnetic Meridian during STEP period: 1 . Preliminary results of low-latitude Pc3's, J. Geomagn. Geoelectr., 44, 261-276, 1992.

Yumoto, K., H. Osaki, K. Fukao, K. Shiokawa, Y. Tanaka, S. I. Solovyev, G. Krymskij, E. F. Vershinin, V. F. Osinin, and $210^{\circ}$ MM Magnetic Observation Group, Correlation of high- and low-latitude Pi2 magnetic pulsations observed at $210^{\circ}$ magnetic meridian chain stations, J. Geomagn. Geoelectr., 46, 925-935, 1994.

Yumoto, K. and the CPMN Group, Characteristics of Pi2 magnetic pulsations observed at the CPMN stations: A review of the STEP results, Earth Planets Space, 53, 981-992, 2001.

Ziesolleck, C. W. S., B. J. Fraser, F. W. Menk, and P. W. McNabb, Spatial characteristics of low-latitude Pc3-4 geomagnetic pulsations, J. Geophys. Res., 98, 197-207, 1993.

M. Nosé (e-mail: nose@kugi.kyoto-u.ac.jp), K. Liou, and P. R. Sutcliffe 